\title{
MicroRNA expression in bone marrow mesenchymal stem cells from mice with steroid-induced osteonecrosis of the femoral head
}

\author{
BINGQING WANG ${ }^{1}$, PENG YU $^{2}$, TAO LI $^{3}$, YANYAN BIAN ${ }^{2}$ and XISHENG WENG ${ }^{2}$ \\ ${ }^{1}$ Fourth Department of Plastic Surgery, Plastic Surgery Hospital Affiliated to The Chinese Academy of Medical Sciences, \\ Peking Union Medical College, Beijing 100144; ${ }^{2}$ Department of Orthopaedic Surgery, Peking Union Medical College \\ Hospital, Peking Union Medical College, Chinese Academy of Medical Science, Beijing 100730; \\ ${ }^{3}$ Department of Orthopaedic Surgery, The Affiliated Hospital of Qingdao University, \\ Qingdao, Shandong 266003, P.R. China
}

Received December 15, 2014; Accepted August 17, 2015

DOI: $10.3892 / \mathrm{mmr} .2015 .4386$

\begin{abstract}
The present study aimed to identify microRNAs (miRNAs) from bone marrow mesenchymal stem cells (BMSCs) in a rat model of steroid-induced osteonecrosis of the femoral head (ONFH) using Affymetrix GeneChip ${ }^{\circledR}$. Following identification of miRNAs, the present study aimed to elucidate the molecular mechanisms underlying steroid-induced ONFH. A total of six C57BL/6J mice were randomly divided into two groups, control and experimental groups ( $\mathrm{n}=3$ per group). The mice in the experimental group were subcutaneously injected with $21 \mathrm{mg} / \mathrm{kg}$ methylprednisolone for 4 weeks, while the mice in the control group were injected with the identical dose of normal saline. The femoral head was subsequently removed and sectioned. Following sectioning, hematoxylin and eosin staining, and terminal deoxynucleotidyl transferase dUTP nick end labeling were performed to confirm the establishment of the model. To replicate the animal model ex vivo, the bone marrow was isolated. Next, different miRNAs were screened for using GeneChip ${ }^{\circledR}$, and the key miRNAs were assessed by bioinformatics analysis and their functions were confirmed. Compared with the control, 23 miRNAs in the experimental group were identified, with seven upregulated and 16 downregulated. Of these miRNAs, putative target miRNAs were predicted by bioinformatics analysis, with two being upregulated (miR-21-3p and miR-652-5p) and five downregulated (miR-206-3p, miR-196a-5p, miR-34b-3p, miR-34c-5p and miR-148a-3p). The results of reverse transcription-quantitative polymerase chain reaction were consistent with the gene-chip results. Steroid-induced ONFH may cause miRNA changes in
\end{abstract}

Correspondence to: Professor Xisheng Weng, Department of Orthopaedic Surgery, Peking Union Medical College Hospital, Peking Union Medical College, Chinese Academy of Medical Science, 1 Shuaifuyuan, Wangfujing, Beijing 100730, P.R. China E-mail: xshweng@medmail.com.cn

Key words: steroid-induced osteonecrosis, femoral head, microRNA, bone marrow mesenchymal stem cell, osteogenesis
BMSCs. Numerous miRNAs regulate osteogenic differentiation and the decrease in miRNA-196a-5p may be important in steroid-induced ONFH.

\section{Introduction}

Glucocorticoid is widely used in the treatment of connective tissue diseases, although large doses may induce osteonecrosis of the femoral head (ONFH) (1). Furthermore, steroid-induced $\mathrm{ONFH}$ can be elevated to the first round of non-traumatic ONFH (2). If the patient fails to obtain effective treatment, the femoral head will collapse and osteoarthritis will become so severe that artificial joint replacement is required (2). Therefore, early diagnosis and treatment of ONFH is required. However, the etiology of the disorder and an effective treatment for ONFH remain to be elucidated. In addition to micro-thrombi caused by micro-circulation disorders leading to local intraosseous hypertension and ischemia (3-6), a disrupted balance between bone necrosis and repair caused by changes in differentiation capacity of bone marrow-derived mesenchymal stem cells (BMSCs) due to glucocorticoids can result in femoral head collapse (7).

MicroRNA (miRNA) is a small, $18-25 \mathrm{nt}$ long single-stranded RNA, . miRNAs are highly conserved in terms sequence and tissue specificity, and they are vital in regulating the function of cells and tissues, and general biological functions (8). Previous studies have revealed that miRNA are vital in regulating the osteogenic differentiation of BMSCs (9). To date, changes in BMSC miRNAs induced by glucocorticoid remain to be fully elucidated. The present study established a mouse model of femoral head necrosis, isolated and cultured mesenchymal stem cells, screened for differences in BMSC miRNAs, and predicted their gene targets using bioinformatics analysis. Additionally, the underlying mechanisms of miRNA regulation in steroid-induced necrosis of the femoral head were investigated to provide powerful evidence for potential clinical treatments.

\section{Materials and methods}

Animals. All experiments were performed following the Guidelines of the Intramural Animal Use and Care Committee 
of the Peking Union Medical College Hospital (Beijing, China). A total of 24 eight-week-old wild-type C57BL/6J mice (Laboratory Animal Center of the Academy of Military Medical Sciences; Beijing, China) were used in the present study. All mice were female, with an average body weight of $18.82 \pm 1.54 \mathrm{~g}$. The animals were housed with a $12 \mathrm{~h}$ light/dark cycle, a constant indoor temperature at $20^{\circ} \mathrm{C}, 48 \%$ humidity and were fed a standard rodent diet. Procedures involving animals and their care were conducted in conformity with NIH guidelines (NIH publication no. 85-23, revised 1996) and was approved by the Animal Care and Use Committee of Peking Union Medical College Hospital.

Animal model of femoral head osteonecrosis. The experimental group of C57BL/6J mice was subcutaneously injected with $21 \mathrm{mg} / \mathrm{kg}$ methylprednisolone (Pfizer, Inc., Ascoli Piceno, Italy) for 4 weeks consecutively, while the control group received an equivalent dose of normal saline. After 4 weeks, the mice were sacrificed (via cervical dislocation following anesthetization with chloral hydrate) and the femurs were removed. The femoral specimens were fixed for $24 \mathrm{~h}$ with $10 \%$ neutral formalin (Guduo Biotechnology Corporation, Shanghai, China; $0.1 \mathrm{mmol} / 1 ; \mathrm{pH} 7.4)$ at room temperature, and subsequently placed in a 10\% EDTA-Tris solution (Thermo Fisher Scientific, Inc., Rockford, IL, USA) at room temperature to decalcify them for 4 weeks (decalcification fluid was changed every 3 days). The femoral heads were considered completely demineralized when the bone was easily pierced with a pin. The samples were dehydrated by a series of graded ethanol washes, placed in xylene (Rongbai Biotechnology, Co., Ltd., Shanghai, China) for $2 \mathrm{~h}$ at room temperature, embedded in paraffin (ToYongBio, Shanghai, China) and sliced into $4-\mu \mathrm{m}$ coronal tissue sections. The tissue sections were processed for hematoxylin and eosin (HE) staining and terminal deoxynucleotidyl transferase dUTP nick end labeling (TUNEL), sealed with neutral resin, and imaged with an inverted phase contrast microscope and camera system (CKX41; Olympus Corporation, Tokyo, Japan).

Isolation, culture, and identification of BMSCs. The mice were sacrificed by cervical dislocation and immersed in $75 \%$ ethanol for $\sim 10 \mathrm{~min}$ at room temperature. The surrounding tissue was peeled away and the long bone was removed from the muscles and connective tissue, and the bone was subsequently washed three times with sterile phosphate-buffered saline (PBS). The metaphysis was removed and the marrow cavity was washed repeatedly using a 1-ml syringe filled with serum-free L-Dulbecco's modified Eagle's medium (DMEM; Gibco Life Technologies, Gaithersburg, MD, USA) to isolate the cells. The samples were uniformly mixed and the cells were seeded into $100-\mathrm{mm}$ culture dishes at a density of $1 \times 10^{6} \mathrm{cells} / \mathrm{ml}$. The isolated BMSCs were cultured for 2 days with L-DMEM, supplemented with 20\% fetal bovine serum (FBS; Gibco Life Technologies), $100 \mathrm{U} / \mathrm{ml}$ penicillin and $100 \mu \mathrm{g} / \mathrm{ml}$ streptomycin (Invitrogen Life Technologies, Carlsbad, CA, USA). The cells were subsequently cultured in L-minimum essential medium, supplemented with $10 \%$ FBS. The mouse mesenchymal stem cell formed adherent colonies after 9-12 days of culture, reaching between 80 and $90 \%$ confluency. The culture media was discarded and the cells were washed three times with PBS, prior to digestion with $0.25 \%$ trypsin and dilution into single cell suspension at a density of $5 \times 10^{6} / \mathrm{ml}$. Aliquots of the cell suspension were added to microcentrifuge tubes (1x10 cells in $200 \mu \mathrm{l} /$ tube) and were subsequently incubated with APC monoclonal rat anti-mouse CD31 (cat. no. 561814; BD Pharmingen, San Diego, CA, USA; 1:250 dilution), FITC monoclonal rat anti-mouse CD34 (cat. no. 560238; BD Pharmingen; 1:250 dilution), monoclonal CD105 FITC (cat. no. ab184667; Abcam, Cambridge, MA, USA; 1:250 dilution), PE monoclonal anti-mouse CD166 (cat. no. 12-1661-81; eBioscience, Inc., San Diego, CA, USA; 1:200 dilution) or control antibodies ( $2 \mu \mathrm{l}$ each). The cells were incubated with the antibody at $4^{\circ} \mathrm{C}$ for $30 \mathrm{~min}$, washed three times with PBS with a centrifugation step in between each wash $\left(4^{\circ} \mathrm{C} ; 380 \mathrm{x} \mathrm{g}\right.$; $5 \mathrm{~min})$. The labeled cells were detected by flow cytometry (FACSCalibur; BD Biosciences, San Jose, CA, USA).

Microarray and data processing. Each experimental and control group consisted of nine mice. The cells from sets of three mice were pooled and cultured together. A micro (mi) RNA microarray was performed in triplicate with the pooled specimens. Primary BMSCs were digested with trypsin and the total RNA was extracted using TRIzol reagent (Invitrogen Life Technologies), according to the manufacturer's instructions. The miRNA and the gene microarrays were performed using a microarray platform (LC Sciences, Houston, TX, USA). The mRNA expression levels were detected using the Affymetrix GeneChip ${ }^{\circledR}$ mouse Genome Array (Affymetrix, Santa Clara, CA, USA). Raw microarray data (CEL files) were normalized using the quantile method (10), and differentially expressed genes were screened using the bioconductor (http://www. bioiconductor.org) Limma package in $\mathrm{R}$ software version 3.0 (Free Software Foundation Inc., Boston, MA, USA). Gene sets with nominal $\mathrm{P}<0.05$ and false discovery rates $<0.25$ were considered to be significant and were included for further investigation.

miRNA-gene network. TargetScan 5.1 (www.targetscan.org) was used in conjunction with miRanda version 2005 (http://www.microrna.org/), PicTar, MirTarget2 and RNAhybrid (http://pictar.mdc-berlin.de/; http://mirdb.org; http://alk.ibms.sinica.edu.tw/cgi-bin/RNAhybrid/RNAhybrid.cgi) to predict the targets of the miRNAs. Genes predicted by any one of the algorithms were considered to be potential targets. To build a miRNA-gene network, the association between miRNAs and genes was assessed based on their differential expression values and according to their interactions in the Sanger miRNA database (http://www.mirbase.org/). An miRNA/gene adjacency matrix $(\mathrm{A}=[\mathrm{ai}, \mathrm{j}])$ was produced, according to the association between the genes and miRNAs, where ai,j represents the weight of the association between gene $i$ and miRNA $j$. In the miRNA-gene network, circles represent genes, squares represent miRNAs and their association is represented by a line. The center of the network is represented by degree, which is the contribution of an miRNA to the surrounding genes or the contribution of a gene to the surrounding miRNAs. The key miRNA and gene in the network always exhibit the highest degree. The network of miRNA-mRNA interactions, representing the critical miRNAs and their targets, was established according to the miRNA degree. 


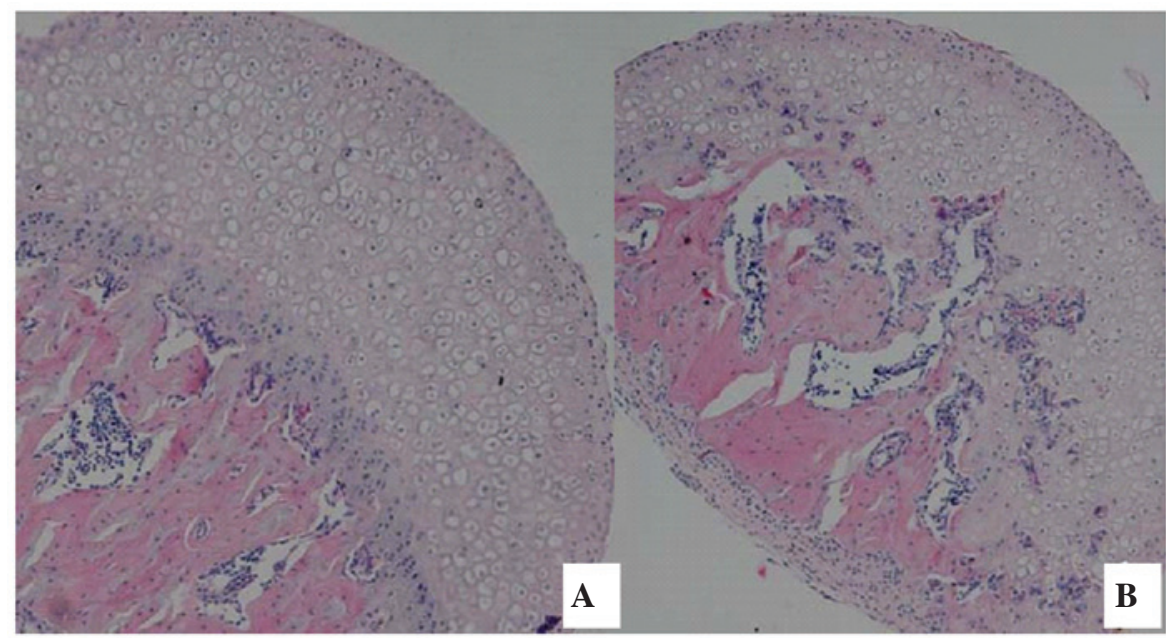

Figure 1. Hematoxylin and eosin staining (magnification, x10) of the femoral head of the C57BL/6J mice. (A) In the control group, the femoral articular surface was smooth and exhibited no defects or collapse. The bone and cartilage cells were normal with good nuclear staining, and the trabecular bone tissue was continuous with no fractures. (B) In the experimental group, the epiphyseal cartilage area is thinner compared with the control, and the epiphysis was perforated as a result of the proliferation of blood vessels and fibrous tissue. Angiogenesis occurred in the middle layer of the epiphyseal cartilage.

A

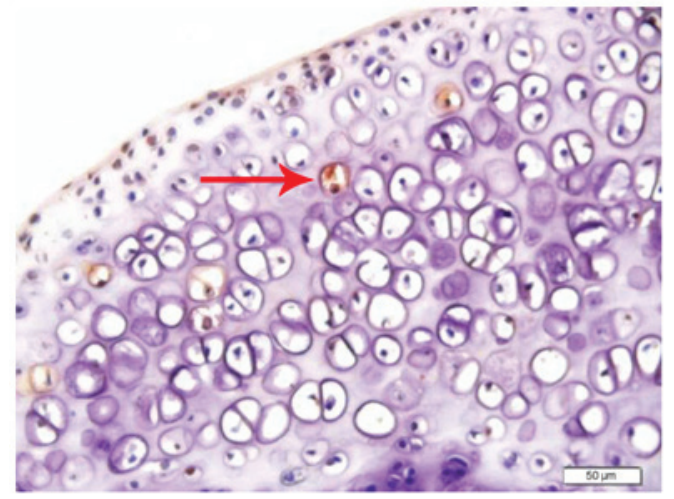

B

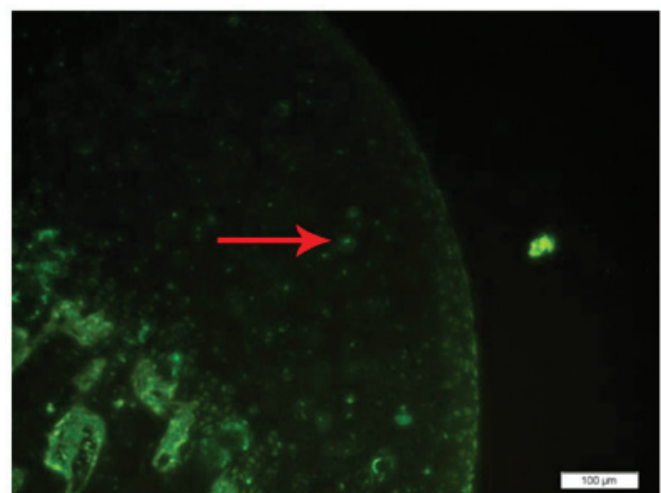

Figure 2. TUNEL and DAB staining. (A) DAB staining was visualized by light microscopy (magnification, x20). The brown nuclear staining (red arrow) indicated necrotic cells. (B) TUNEL staining was observed by fluorescence microscopy (magnification, x10). Fluorescent signal (red arrow) indicated necrotic cells. TUNEL, terminal deoxynucleotidyl transferase dUTP nick-end labeling; DAB, 3,3'-diaminobenzidine.

Reverse transcription-quantitative polymerase chain reaction (RT-qPCR) of miRNA. Differentially expressed miRNAs were selected for validation, including miR-21-3p, miR-652-5p, miR-206-3p, miR-196a-5p, miR-34b-3p, miR-34c-5p and miR-148a-3p. The expression of these mature miRNAs was determined using stem-loop RT, followed by PCR analysis, as previously described (11). The PCR was performed in triplicate for each sample and U6 served as a positive control for miRNA PCR. The miRNAs levels were quantified using mouse TaqMan MicroRNA assays (Applied Biosystems Life Technologies, Beijing, China). PCR was conducted using a thermocycler (ABI 7900HT; Applied Biosystems Life Technologies) for 35 cycles and all reagents were obtained from Applied Biosystems Life Technologies. The stem-loop primers used are presented in Table I. The relative quantity of miRNA was normalized against U6 snRNA and the fold-change for each miRNA was calculated using the $2^{-\Delta \Delta C t}$ method. The relative expression values of each miRNA were calculated using the $2^{-\Delta \Delta \mathrm{Ct}}$ method, as follows: $\Delta \mathrm{Ct}=\mathrm{Ct}$ (each miRNA) - Ct (U6). The fold changes of the miRNA expression values in the experimental group samples versus normal controls were determined by the $2^{-\Delta \Delta \mathrm{Ct}}$ method, as follows: $\Delta \Delta \mathrm{Ct}=$ median $\Delta \mathrm{Ct}_{\text {experimental group }}-$ median $\Delta \mathrm{Ct}_{\text {control group }}$.

\section{Results}

HE staining and TUNEL. Using HE staining, it was demonstrated that, compared with the control group samples (Fig. 1A), the experimental group samples (Fig. 1B) exhibited a thinner epiphyseal cartilage zone in the femoral head. However, while the proliferative zone and cartilage zone were easily distinguished, the epiphysis exceeded normal parameters and early trabecular fractures were observed. In the region containing epiphyseal cartilage, vascular proliferation and fibrous tissue growth was observed, multiple small necrotic foci were observed near the femoral epiphyseal line and the femoral articular surface was not smooth, indicating significant wear to the tissue.

TUNEL revealed that there were no apoptotic cells in the control group, while various examples of apoptotic osteocytes and osteoblasts were observed in the experimental group (Fig. 2). 
Table I. Stem-loop primer sequences used in the present study.

\begin{tabular}{ll}
\hline MicroRnA & \multicolumn{1}{c}{ Primer sequence } \\
\hline mmu-miR-21-3p & UGUACCACCUUGUCGGAUAGCUUAUCAGACUGAUGUUGACUGUUGAAUCUCAUGGCAAC \\
AGCAGUCGAUGGGCUGUCUGACAUUUUGGUAUC & AGGAACAGCUAUGUACUGCACAACCCUAGGAGGGGGUGCCAUUCACAUAGAGUAUAAUU \\
mmu-miR-652-5p & GAAUGGCGCCACUAGGGUUGUGCAGUGUACAGCCUACAC \\
mmu-miR-206-3p & CCAGGCCACAUGCUUCUUUAUAUCCUCAUAGAUAUCUCAGCACUAUGGAAUGUAAGGAA \\
mmu-miR-34b-3p & GUGCUCGGUUUGUAGGCAGUGUAAUUAGCUGAUUGUAGUGCGGUGCUGACAAUCACUAA \\
& CUCCACUGCCAUCAAAACAAGGCAC \\
mmu-miR-196a-5p & AGCUGAUCUGUGGCUUAGGUAGUUUCAUGUUGUUGGGAUUGAGUUUUGAACUCGGCAA \\
& CAAGAAACUGCCUGAGUUACAUCAGUC \\
mmu-miR-34c-5p & AGUCUAGUUACUAGGCAGUGUAGUUAGCUGAUUGCUAAUAGUACCAAUCACUAACCACA \\
& CAGCCAGGUAAAAAGACU \\
mmu-miR-148a-3p & AGCCAGUUUGGUCUUUUGAGACAAAGUUCUGAGACACUCCGACUCUGAGUAUGAUAGAA \\
& GUCAGUGCACUACAGAACUUUGUCUCUAGAGGCUGUGGUC
\end{tabular}

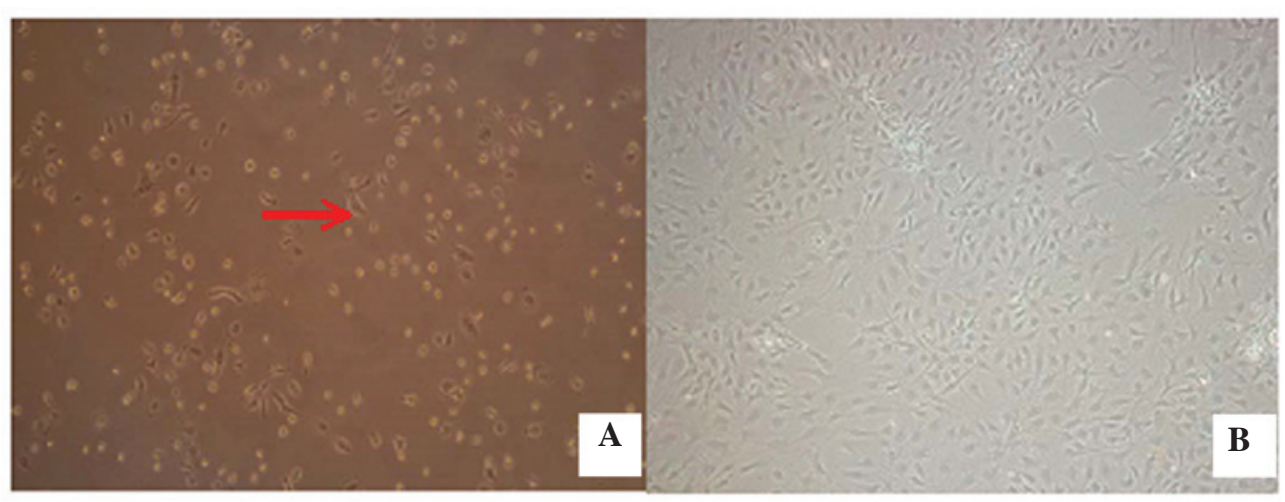

Figure 3. BMSCs were observed by light microscopy (magnification, x20). (A) Immediately following the first media change after $48 \mathrm{~h}$, a small number of fusiform adherent cells were visible. (B) Following culturing for 9 days, the cells had markedly proliferated and taken on a uniform, spindle-like morphology. BMSC, bone marrow mesenchymal stem cells.
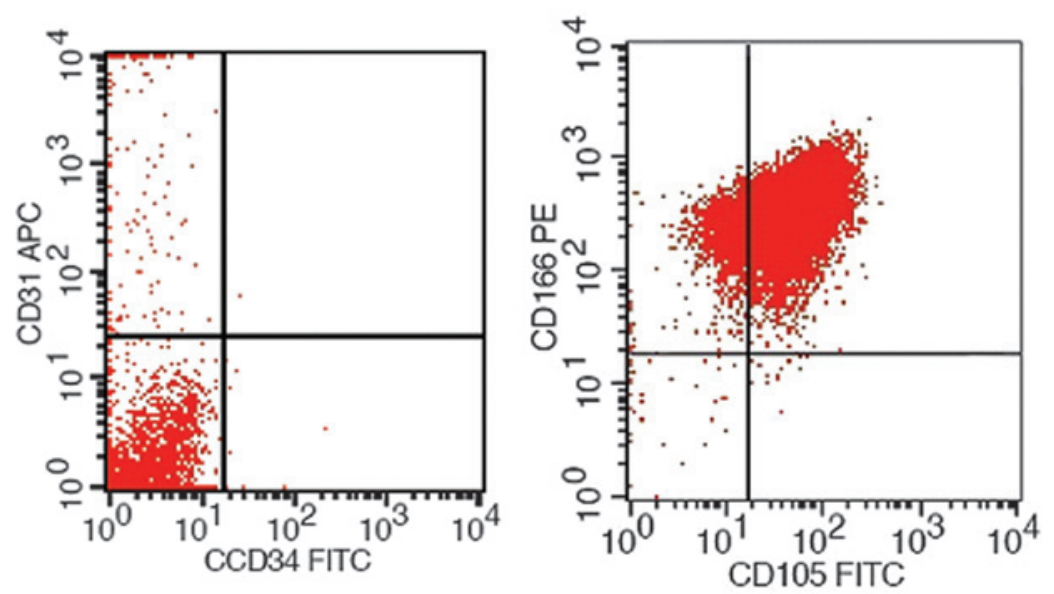

Figure 4. Flow cytometric analysis of the surface antigen profiles of bone marrow mesenchymal stem cell. FITC, fluorescein isothiocyanate; $\mathrm{CD}$, cluster of differentiation; APC, allophycocyanin; PE, Phycoerythrin.

Culture of BMSCs in vitro. Following the initial exchange of the culture medium after $48 \mathrm{~h}$, a small number of adherent cells, which were small and round, with a quiescent phenotype were observed (Fig. 3). As the incubation duration increased, these cells rapidly proliferated and reached $80-90 \%$ confluency by 9 days. The cells exhibited a long, 
Table II. Differentially expressed miRs identified in bone marrow stromal cells.

\begin{tabular}{|c|c|c|c|c|}
\hline $\operatorname{miR}$ & ONFH mean & Control mean & Fold-change & P-value \\
\hline \multicolumn{5}{|c|}{ Upregulated microRNAs } \\
\hline mmu-miR-500-3p & 202.62 & 99.04 & 2.05 & $4.1 \times 10^{-4}$ \\
\hline mmu-miR-21-3p & 13.40 & 6.31 & 2.13 & $1.9 \times 10^{-3}$ \\
\hline mmu-miR-342-5p & 171.47 & 91.76 & 1.87 & $4.7 \times 10^{-3}$ \\
\hline mmu-miR-92b-5p & 12.49 & 3.04 & 4.11 & $9.9 \times 10^{-3}$ \\
\hline mmu-miR-720 & 34.55 & 19.34 & 1.79 & $2.1 \times 10^{-2}$ \\
\hline mmu-miR-652-5p & 11.62 & 4.29 & 2.71 & $4.0 \times 10^{-2}$ \\
\hline mmu-miR-5112 & 26.21 & 212.02 & 2.01 & $4.3 \times 10^{-2}$ \\
\hline \multicolumn{5}{|c|}{ Downregulated microRNAs } \\
\hline mmu-miR-206-3p & 2.11 & 162.65 & 0.013 & $1.0 \times 10^{-7}$ \\
\hline mmu-miR-30a-3p & 7.41 & 18.36 & 0.40 & $1.3 \times 10^{-3}$ \\
\hline mmu-miR-127-3p & 12.32 & 26.12 & 0.47 & $1.7 \times 10^{-3}$ \\
\hline mmu-miR-34b-3p & 21.38 & 55.84 & 0.38 & $2.3 \times 10^{-3}$ \\
\hline mmu-miR-345-5p & 3.37 & 8.30 & 0.41 & $2.4 \times 10^{-3}$ \\
\hline mmu-miR-615-3p & 7.62 & 20.25 & 0.38 & $6.6 \times 10^{-3}$ \\
\hline mmu-miR-345-3p & 2.38 & 5.50 & 0.43 & $8.3 \times 10^{-3}$ \\
\hline mmu-miR-1906 & 6.15 & 12.06 & 0.51 & $1.3 \times 10^{-2}$ \\
\hline mmu-miR-196a-5p & 30.17 & 64.42 & 0.47 & $1.4 \times 10^{-2}$ \\
\hline mmu-miR-665-3p & 2.00 & 4.66 & 0.43 & $1.7 \times 10^{-2}$ \\
\hline mmu-miR-669p-3p & 2.00 & 3.52 & 0.57 & $1.7 \times 10^{-2}$ \\
\hline mmu-miR-3096-3p & 9.57 & 18.18 & 0.53 & $2.6 \times 10^{-2}$ \\
\hline mmu-miR-382-5p & 2.13 & 4.52 & 0.47 & $2.8 \times 10^{-2}$ \\
\hline mmu-miR-467a-5p & 3.27 & 6.87 & 0.48 & $3.0 \times 10^{-2}$ \\
\hline mmu-miR-34c-5p & 11.35 & 22.74 & 0.50 & $4.2 \times 10^{-2}$ \\
\hline mmu-miR-148a-3p & 2.30 & 3.72 & 0.62 & $4.9 \times 10^{-2}$ \\
\hline
\end{tabular}

miRs identified in ONFH and control BMSCs, with mean expression values calculated following global normalization and statistical analysis using Student's t-test. Fold-increase in ONFH BMSCs compared with the control BMSCs is shown, with the threshold set at 1.5-fold expression relative to the controls $(\mathrm{P}<0.05)$. Fold-decrease in ONFH BMSCs compared with control BMSCs is shown, with the threshold set at 0.67 -fold expression relative to the controls $(\mathrm{P}<0.05)$. ONFH, osteonecrosis of the femoral head; BMSCs, bone marrow stromal cells; miR, microRNA.

Table III. miRNAs associated with osteogenic differentiation.

\begin{tabular}{|c|c|c|c|c|}
\hline $\operatorname{miR}$ & ONFH mean & Control mean & Fold-change & P-value \\
\hline \multicolumn{5}{|l|}{ Upregulated miRNAs } \\
\hline mmu-miR-21-3p & 13.40 & 6.31 & 2.13 & $1.9 \times 10^{-3}$ \\
\hline mmu-miR-652-5p & 11.62 & 4.29 & 2.71 & $4.0 \times 10^{-2}$ \\
\hline \multicolumn{5}{|c|}{ Downregulated miRNAs } \\
\hline mmu-miR-206-3p & 2.11 & 162.65 & 0.01 & $1.0 \times 10^{-7}$ \\
\hline mmu-miR-34b-3p & 21.38 & 55.84 & 0.38 & $2.3 \times 10^{-3}$ \\
\hline mmu-miR-196a-5p & 30.17 & 64.42 & 0.47 & $1.4 \times 10^{-2}$ \\
\hline mmu-miR-34c-5p & 11.35 & 22.74 & 0.50 & $4.2 \times 10^{-2}$ \\
\hline mmu-miR-148a-3p & 2.30 & 3.72 & 0.62 & $4.9 \times 10^{-2}$ \\
\hline
\end{tabular}

miR, microRNA; ONFH, osteonecrosis of the femoral head.

spindle-like morphology, with a small quantity of protrusion formation, which indicated fibroblast-like growth at 9 days (Fig. 3).
BMSC surface antigen tests. BMSCs were identified by flow cytometry, the results of which are shown in Fig. 4. Isolated and cultured BMSCs expressed surface antigens that were 


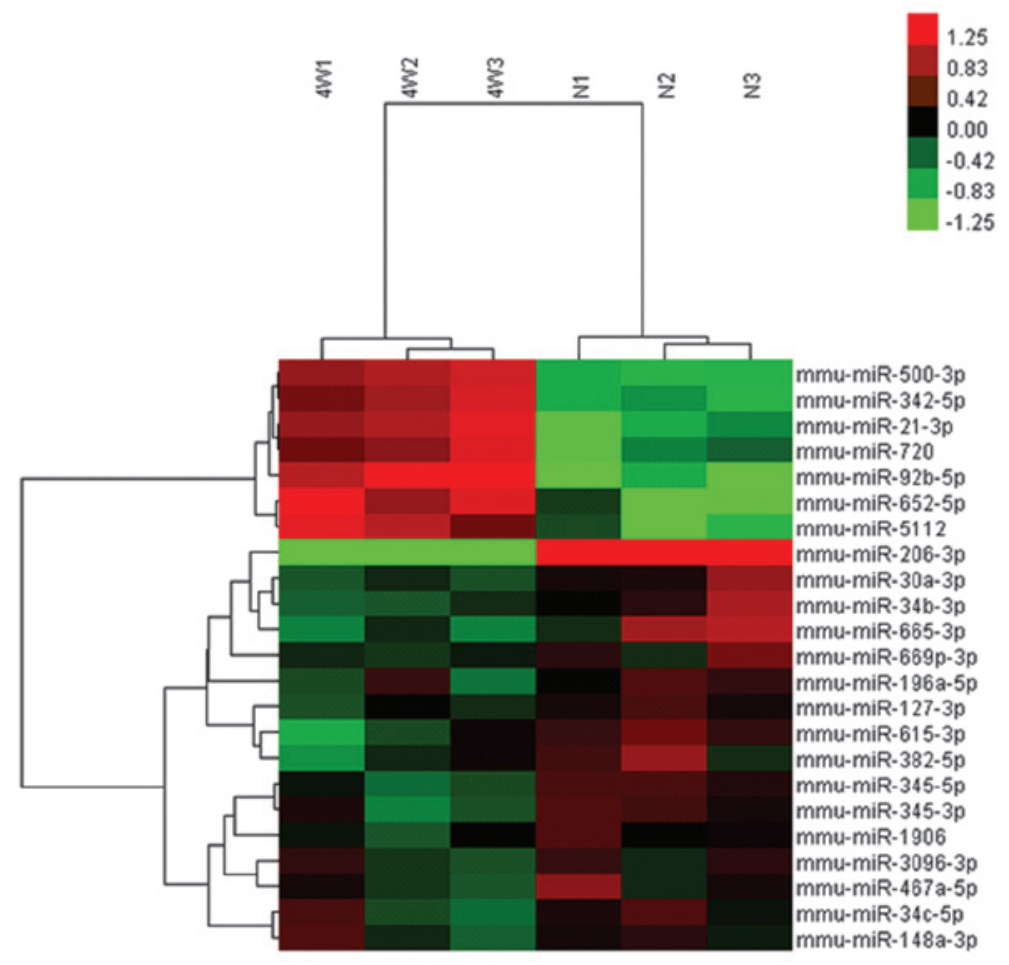

Figure 5. miRNA profiles differentiate ONFH BMSCs from control BMSCs. The samples consisted of paired samples from nine mice. The downregulated (green) and upregulated (red) miRNAs were identified in hematopoietic stem cells. $\mathrm{P}<0.01$ denoted statistically significant differences between the ONFH and control BMSCs. ONFH, osteonecrosis of the femoral head; BMSC, bone marrow mesechymal stem cells; miRNA, microRNA.

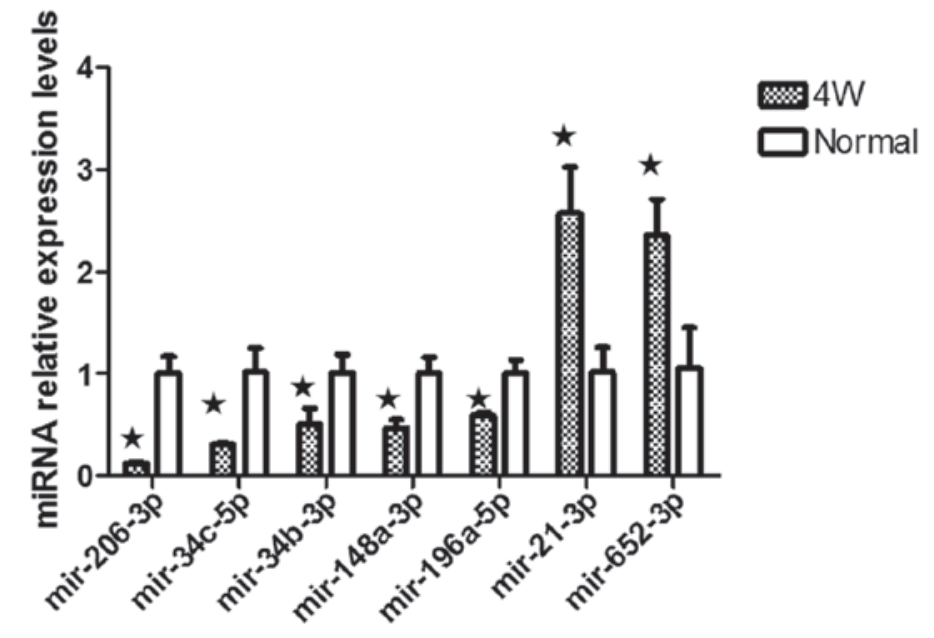

Figure 6. Reverse transcription-quantitative polymerase chain reaction confirmation of differentially expressed miRNAs. The results were generally consistent with the microarray results. ${ }^{\mathrm{P}}<0.05$. miR, microRNA; miRNA, microRNA.

comparable to those that were expressed by stem cells, including high expression levels of CD105 and CD166, and negative expression of CD31 and CD34. Therefore, based on the present study, it was identified that these cells were BMSCs.

miRNA expression profiles of the BMSCs. Using the Affymetrix GeneChip ${ }^{\circledR}$ mouse Genome Array, the miRNA expression profiles of the BMSCs in the control and experimental groups were determined. A total of seven BMSC miRNAs were upregulated $>1.5$-fold in the experimental group compared with the controls, while 16 miRNAs were expressed below the threshold level (0.67-fold; Table II; Fig. 5). RT-qPCR confirmed the upregulated expression of the seven miRNAs, confirming the results from the microarray assay (Fig. 6).

Microarray-based analysis. The global expression of miRNAs and mRNAs in ONFH BMSCs was investigated using microarray technologies. Following miRNA microarray data pre-processing, differential expression of 23 miRNAs was identified, of which, seven were upregulated and 16 were downregulated. The microarray results were further analyzed using the Targetscan database and bioinformatics, and determined that miR-21-3p, miR-652-5p, miR-206-3p, miR-196a-5p, miR-34b-3p, miR-34c-5p and miR-148a-3p may be involved 
in osteogenic differentiation (Table III). To confirm the microarray results, seven miRNAs, miR-21-3p, miR-652-5p, miR-206-3p, miR-196a-5p, miR-34b-3p, miR-34c-5p and miR-148a-3p, were analyzed by RT-qPCR (Fig. 6).

\section{Discussion}

The pathological process underlying the development of femoral head avascular necrosis is highly complicated. A variety of factors can cause the death of bone cells and marrow, and this death and subsequent repair may lead to structural changes in the femoral head, femoral head collapse, and/or joint dysfunction. These symptoms are hallmarks of ONFH. Hormones are the leading cause of avascular necrosis of the femoral head (1). In the United States, 12 million patients suffer from steroid-induced femoral head necrosis annually (2). The mechanisms underlying glucocorticoid-induced femoral head necrosis are multifaceted. For example, long-term usage of large doses of glucocorticoid can lead to adipogenesis/fat hypertrophy (3), endothelial cell dysfunction or damage (4), microthrombus formation (5) and high intraosseous pressure (6). These, in turn, can eventually cause damage to the vascular endothelium, microcirculation dysfunction and decreased arterial blood flow. Eventually, bone ischemia, hypoxia, necrosis, damage to bone structure and function, and avascular necrosis of the femoral head may occur (12). A previous study focused on the effects of steroids on local hemodynamic aspects of the femoral head, which lead to ONFH (13), and another previous report revealed that BMSCs exert an important role in femoral head necrosis. Wang et al (14) and others cultured BMSCs isolated from patients with steroid-induced osteonecrosis and revealed that the efficiency of colony formation was significantly lower compared with that observed in the healthy controls, a result, which demonstrated reduced activity of BMSCs in patients with femoral head necrosis. Previous studies have demonstrated that large doses of corticosteroids can lead to decreased expression of Runx2/Cbfa1 in BMSCs, while under identical conditions the expression of PPAR- $\gamma$ and Dickkopf-1 increased. These changes in the expression levels affected the differentiation of BMSCs and led to imbalances in bone resorption and calcaneus destruction $(15,16)$. Based on these previous studies, there appears to be a close association was observed between BMSCs and femoral head necrosis, therefore, making the use of BMSCs in the treatment of osteonecrosis a topic of significant interest.

There have been no direct reports demonstrating that changes in miRNA expression can result in femoral head necrosis; however, a large number of studies have demonstrated that miRNA is important in regulating the osteogenic differentiation of BMSCs. A previous study knocked out Dicer and Drosha, critical proteins in the miRNA pathway, and revealed that mesenchymal stem cells were unable to develop into osteoblasts and adipocytes (17). During this investigation of the BMP pathway, it was revealed that miRNA-208 (18), miRNA-125b (19), miRNA-141 (20) and miRNA-200a (20) promoted osteoblast differentiation. Additionally, investigations on the Wnt pathway revealed that miRNA-27 (21), miRNA-29a (22), and miRNA-29b (23) promoted osteoblast differentiation. Therefore, it was speculated that large doses of hormones affect the osteogenic and adipogenic differentiation of BMSCs by altering the miRNA expression levels, destroying the balance between osteogenesis and osteoclast activity, and ultimately leading to necrosis of the femoral head.

The present study investigated murine BMSCs in an attempt to elucidate the pathogenesis of steroid-induced osteonecrosis through the comparison of miRNA expression levels between these cells isolated from ONFH mice and controls. Following analysis of the microarray results, 23 significant differences were identified in the miRNA expression between the ONFH group and the control group, with seven upregulated and 16 downregulated miRNAs. Furthermore, through bioinformatics analysis, it was determined that seven miRNAs (miR-21-3p, miR-652-5p, miR-206-3p, miR-196a-5p, miR-34b-3p, miR-34c-5p and miR-148a-3p) may be involved in osteogenic differentiation. Of these, two were upregulated (miR-21-3p and miR-652-5p), and five were downregulated (miR-206-3p, miR-196a-5p, miR-34b-3p, miR-34c-5p and miR-148a-3p). The expression of miR-206-3p decreased markedly compared with the control group. Previous studies have suggested that miRNA-206-3p can inhibit IdI-3/MyoR, thereby activating MyoD and promoting muscle differentiation $(24,25)$. During the process of osteoblast differentiation, miRNA-206-3p expression levels are known to decrease (26). The target of miR-196a-5p is the Hox gene family, which is important in animal limb development $(27,28)$ and BMSC differentiation. Additionally, miRNA-196a-5p promotes the osteogenic differentiation via the BMP pathway. miRNA-34b-3p and miR-34c-5p are involved in the Notch (29), Runx2 (30) and SATB2 pathways (31), and are important in osteoblast differentiation by inhibiting osteogenic differentiation. Following an investigation of osteoporosis, Yang et al (32) revealed that miRNA-21-3p inhibits Spry-1 and therefore, promotes osteoblast differentiation $(32,33)$, whereas another previous study revealed that increased levels of miRNA-21-3p and miRNA-148a-3p are important in osteoclast differentiation $(34,35)$. Additionally, it has been demonstrated that miRNA-652-5p expression increases in osteosarcoma (36). From these previous results, it was concluded that osteogenic and adipogenic differentiation of BMSCs is regulated by multiple miRNAs, and requires an integrated signaling network. Large doses of hormones can affect the differentiation of BMSCs by altering the expression of miRNAs, causing imbalances between osteogenesis and osteoclast activity, leading to the occurrence of osteonecrosis.

According to previous studies, the downregulation of miR-206-3p, miR-34b-3p and miR-34c-5p, as well as the upregulation of miR-21-3p, has a role in promoting osteogenic differentiation. By contrast, the downregulation of miR-196a-5p inhibits osteogenic differentiation. Furthermore, based on these previous studies, there is a close association exists between osteogenic differentiation of BMSCs and femoral head necrosis in mice. The present study therefore hypothesized that miR-196a-5p may be important in the process of steroid-induced femoral head necrosis. The underlying mechanisms remain to be elucidated in further studies.

\section{References}

1. Fukushima W, Fujioka M, Kubo T, Tamakoshi A, Nagai M and Hirota Y: Nationwide epidemiologic survey of idiopathic osteonecrosis of the femoral head. Clin Orthop Relat Res 468: 2715-2724, 2010 
2. Koo KH, Kim R, Kim YS, Ahn IO, Cho SH, Song HR, Park YS, Kim $\mathrm{H}$ and Wang GJ: Risk period for developing osteonecrosis of the femoral head in patients on steroid treatment. Clin Rheumatol 21: 299-303, 2002.

3. Motomura G, Yamamoto T, Miyanishi K, Yamashita A, Sueishi K and Iwamoto Y: Bone marrow fat-cell enlargement in early steroid-induced osteonecrosis-a histomorphometric study of autopsy cases. Pathol Res Pract 200: 807-811, 2005.

4. Wei J, Shi Y, Zheng L, Zhou B, Inose H, Wang J, Guo XE, Grosschedl R and Karsenty G: miR-34 s inhibit osteoblast proliferation and differentiation in the mouse by targeting SATB2. J Cell Biol 197: 509-521, 2012.

5. Li M, Yu M, Liu C, Zhu H, He X, Peng S and Hua J: miR-34c works downstream of p53 leading to dairy goat male germline stem-cell (mGSCs) apoptosis. Cell Prolif 46: 223-231, 2013.

6. Yang N, Wang G, Hu C, Shi Y, Liao L, Shi S, Cai Y, Cheng S, Wang X, Liu Y, et al: Tumor necrosis factor $\alpha$ suppresses the mesenchymal stem cell osteogenesis promoter miR-21 in estrogen deficiency-induced osteoporosis. J Bone Miner Res 28: 559-573, 2013

7. Tan G, Kang PD and Pei FX: Glucocorticoids affect the metabolism of bone marrow stromal cells and lead to osteonecrosis of the femoral head: a review. Chin Med J (Engl) 125: 134-139. 2012.

8. He X, Yan YL, Eberhart JK, Herpin A, Wagner TU, Schartl M and Postlethwait JH: miR-196 regulates axial patterning and pectoral appendage initiation. Dev Biol 357: 463-477, 2011.

9. Oskowitz AZ, Lu J, Penfornis P, Ylostalo J, McBride J, Flemington EK, Prockop DJ and Pochampally R: Human multipotent stromal cells from bone marrow and microRNA: regulation of differentiation and leukemia inhibitory factor expression. Proc Natl Acad Sci USA 105: 18372-18377, 2008.

10. BM Bolstad,Irizarry RA, Astrand M and Speed TP: A comparison of normalization methods for high density oligonucleotide array data based on variance and bias. Bioinformatics 19: 185-193, 2003.

11. Chen C, Ridzon DA, Broomer AJ, Zhou Z, Lee DH, Nguyen JT, Barbisin M, Xu NL, Mahuvakar VR, Andersen MR, et al: Real-time quantification of microRNAs by stem-loop RT-PCR. Nucleic Acids Res 33: e179, 2005.

12. Lian JB, Stein GS, van Wijnen AJ, Stein JL, Hassan MQ, Gaur T and Zhang Y: MicroRNA control of bone formation and homeostasis. Nat Rev Endocrinol 8: 212-227, 2012.

13. Kerachian MA, Séguin C and Harvey EJ: Glucocorticoids in osteonecrosis of the femoral head: A new understanding of the mechanisms of action. J Steroid Biochem Mol Biol 114: 121-128, 2009.

14. Wang BL, Sun W, Shi ZC, Lou JN, Zhang NF, Shi SH, Guo WS, Cheng LM, Ye LY, Zhang WJ and Li ZR: Decreased proliferation of mesenchymal stem cells in corticosteroid-induced osteonecrosis of femoral head. Orthopedics 31: 444, 2008

15. Hornstein E, Mansfield JH, Yekta S, Hu JK, Harfe BD, McManus MT, Baskerville S, Bartel DP and Tabin CJ: The microRNA miR-196 acts upstream of Hoxb8 and Shh in limb development. Nature 438: 671-674, 2005.

16. Choo SW and Russell S: Genomic approaches to understanding Hox gene function. Adv Genet 76: 55-91, 2011.

17. Oskowitz AZ, Lu J, Penfornis P, Ylostalo J, McBride J, Flemington EK, Prockop DJ and Pochampally R: Human multipotent stromal cells from bone marrow and microRNA: Regulation of differentiation and leukemia inhibitory factor expression. Proc Natl Acad Sci USA 105: 18372-18377, 2008.

18. Itoh T, Takeda S and Akao Y: MicroRNA-208 modulates BMP-2-stimulated mouse preosteoblast differentiation by directly targeting V-ets erythroblastosis virus E26 oncogene homolog 1. J Biol Chem 285: 27745-27752, 2010.
19. Mizuno Y, Yagi K, Tokuzawa Y, Kanesaki-Yatsuka Y, Suda T, Katagiri T, Fukuda T, Maruyama M, Okuda A, Amemiya T, et al: miR-125b inhibits osteoblastic differentiation by down-regulation of cell proliferation. Biochem Biophys Res Commun 368: 267-272, 2008.

20. Itoh T, Nozawa Y and Akao Y: MicroRNA-141 and -200a are involved in bone morphogenetic protein-2-induced mouse pre-osteoblast differentiation by targeting distal-less homeobox 5. J Biol Chem 284: 19272-19279, 2009.

21. Wang $\mathrm{T}$ and $\mathrm{Xu} \mathrm{Z}$ : miR-27 promotes osteoblast differentiation by modulating Wnt signaling. Biochem Biophys Res Commun 402: 186-189, 2010.

22. Kapinas K, Kessler C, Ricks T, Gronowicz G and Delany AM: miR-29 modulates Wnt signaling in human osteoblasts through a positive feedback loop. J Biol Chem 285: 25221-25231, 2010.

23. Li Z, Hassan MQ, Jafferji M, Aqeilan RI, Garzon R, Croce CM, van Wijnen AJ, Stein JL, Stein GS and Lian JB: Biological functions of miR-29b contribute to positive regulation of osteoblast differentiation. J Biol Chem 284: 15676-15684, 2009.

24. Kim HK, Lee YS, Sivaprasad U, Malhotra A and Dutta A Muscle-specific microRNA miR-206 promotes muscle differentiation. J Cell Biol 174: 677-687, 2006.

25. Luo W, Nie Q and Zhang X: MicroRNAs involved in skeletal muscle differentiation. J Genet Genomics 40: 107-116, 2013.

26. Inose H, Ochi H, Kimura A, Fujita K, Xu R, Sato S, Iwasaki M, Sunamura S, Takeuchi Y, Fukumoto S, et al: A microRNA regulatory mechanism of osteoblast differentiation. Proc Natl Acad Sci USA 106: 20794-20799, 2009.

27. He X, Yan YL, Eberhart JK, Herpin A, Wagner TU, Schartl M and Postlethwait JH: miR-196 regulates axial patterning and pectoral appendage initiation. Dev Biol 357: 463-477, 2011.

28. Sehm T, Sachse C, Frenzel C and Echeverri K: miR-196 is an essential early-stage regulator of tail regeneration, upstream of key spinal cord patterning events. Dev Biol 334: 468-480, 2009.

29. Bae Y, Yang T, Zeng HC, Campeau PM, Chen Y, Bertin T, Dawson BC, Munivez E, Tao J and Lee BH: miRNA-34c regulates Notch signaling during bone development. Hum Mol Genet 21: 2991-3000, 2012.

30. Zhang Y, Xie RL, Croce CM, Stein JL, Lian JB, van Wijnen AJ and Stein GS: A program of microRNAs controls osteogenic lineage progression by targeting transcription factor Runx2. Proc Natl Acad Sci USA 108: 9863-9868, 2011.

31. Wei J, Shi Y, Zheng L, Zhou B, Inose H, Wang J, Guo XE, Grosschedl R and Karsenty G: miR-34s inhibit osteoblast proliferation and differentiation in the mouse by targeting SATB2. J Cell Biol 197: 509-521, 2012.

32. Yang N, Wang G, Hu C, Shi Y, Liao L, Shi S, Cai Y, Cheng S, Wang X, Liu Y, et al: Tumor necrosis factor $\alpha$ suppresses the mesenchymal stem cell osteogenesis promoter miR-21 in estrogen deficiency-induced osteoporosis. J Bone Miner Res 28: 559-573, 2013.

33. Lian JB, Stein GS, van Wijnen AJ, Stein JL, Hassan MQ, Gaur T and Zhang Y: MicroRNA control of bone formation and homeostasis. Nat Rev Endocrinol 8: 212-227, 2012.

34. Cheng P, Chen C, He HB, Hu R, Zhou HD, Xie H, Zhu W, Dai RC, Wu XP, Liao EY and Luo XH: miR-148a regulates osteoclastogenesis by targeting V-maf musculoaponeurotic fibrosarcoma oncogene homolog B. J Bone Miner Res 28: 1180-1190, 2013.

35. Sugatani T, Vacher J and Hruska KA: A microRNA expression signature of osteoclastogenesis. Blood 117: 3648-3657, 2011.

36. Lulla RR, Costa FF, Bischof JM, Chou PM, de F Bonaldo M, Vanin EF and Soares MB: Identification of differentially expressed MicroRNAs in Osteosarcoma. Sarcoma 2011: 732690, 2011. 